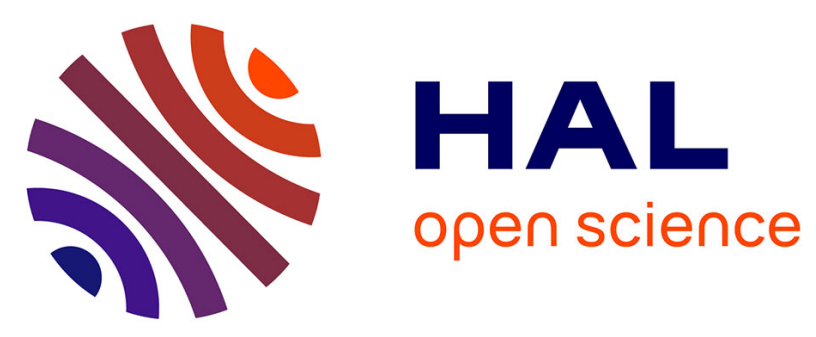

\title{
Crystal structures of two camelid nanobodies raised against GldL, a component of the Type IX Secretion System from Flavobacterium johnsoniae
}

Thi Trang Nhung, Anaïs Gaubert, Pauline Melani, Christian Cambillau, Alain Roussel, Philippe Leone

\section{To cite this version:}

Thi Trang Nhung, Anaïs Gaubert, Pauline Melani, Christian Cambillau, Alain Roussel, et al.. Crystal structures of two camelid nanobodies raised against GldL, a component of the Type IX Secretion System from Flavobacterium johnsoniae. Acta crystallographica Section F : Structural biology communications [2014-..], 2021. hal-03358191

\section{HAL Id: hal-03358191 \\ https://hal.science/hal-03358191}

Submitted on 29 Sep 2021

HAL is a multi-disciplinary open access archive for the deposit and dissemination of scientific research documents, whether they are published or not. The documents may come from teaching and research institutions in France or abroad, or from public or private research centers.
L'archive ouverte pluridisciplinaire HAL, est destinée au dépôt et à la diffusion de documents scientifiques de niveau recherche, publiés ou non, émanant des établissements d'enseignement et de recherche français ou étrangers, des laboratoires publics ou privés. 


\section{Crystal structures of two camelid nanobodies raised against GIdL, a component of the Type IX Secretion System from Flavobacterium johnsoniae.}

Thi Trang Nhung Trinh ${ }^{\text {ab }}$, Anaïs Gaubert ${ }^{\mathrm{cd}}$, Pauline Melani ${ }^{\mathrm{cd}}$, Christian Cambillau ${ }^{\mathrm{cd}}$, Alain Roussel $^{\text {cd }}$ and Philippe Leone ${ }^{\text {cd } *}$

${ }^{a}$ Faculty of Medical Technology, PHENIKAA University, Yen Nghia, Ha Dong, Hanoi 12116, Vietnam

${ }^{\mathrm{b}}$ PHENIKAA Research and Technology Institute (PRATI), A\&A Green Phoenix Group JSC, No. 167 Hoang Ngan, Trung Hoa, Cau Giay, Hanoi 11313, VietNam

${ }^{\mathrm{c}}$ Centre National de la Recherche Scientifique, Architecture et Fonction des Macromolécules Biologiques, UMR 7257, Marseille, France

dAix-Marseille Université, Architecture et Fonction des Macromolécules Biologiques, UMR 7257, Marseille, France

* Correspondence email: Philippe.Leone@afmb.univ-mrs.fr

Synopsis Two nanobodies raised against the GldL cytoplasmic domain were produced, the interaction with their target was characterized, and their crystal structures were solved.

Abstract GldL is an inner-membrane protein essential for the function of the type IX secretion system (T9SS) from Flavobacterium johnsoniae. The complex it forms with GldM is supposed to act as a new rotary motor involved in the gliding motility of the bacteria. In the context of structural studies of GldL to gain information on T9SS assembly and function, two camelid nanobodies were selected, produced and purified. Their interaction with the GldL cytoplasmic domain was characterized, and their crystal structures were solved. These nanobodies will be used as crystallization chaperones to help for 
crystallization of the GldL cytoplasmic domain and could help as well to solve the structure of the complex by molecular replacement.

\section{Keywords: nanobody; type IX secretion system; interaction; domain-swapping}

\section{Introduction}

The type IX secretion system (T9SS) is a sophisticated machinery that spans both the inner and outer membranes. T9SS is widespread in Fibrobacteres-Chlorobi-Bacteroidetes superphylum (CFB group) (Veith et al., 2017) and can deliver various substrates through the cell envelope. These substrate proteins are secreted by a two-step mechanism: they are first exported into the periplasm by the Sec pathway after cleavage of their $\mathrm{N}$-terminal signal peptide, then their conserved $\mathrm{C}$-terminal domain (CTD) triggers their secretion outside the cell by the T9SS. The T9SS has been mainly studied in the two bacterial models Porphyromonas gingivalis and Flavobacterium johnsoniae. In P. gingivalis, a human oral pathogen involved in periodontal diseases, the T9SS secretes virulence factors associated with pathogenicity. In F. johnsoniae, a non-pathogenic soil bacterium that moves rapidly on solid surfaces by gliding motility, the T9SS secretes adhesins involved in the attachment of the cell to the surface. To date, 18 genes have been identified as essential for T9SS function in P. gingivalis (Lasica et al., 2017), and 12 in F. johnsoniae (McBride \& Zhu, 2013). Four of these genes, porK-porL-porM-porN in $P$. gingivalis, and gldK-gldL-gldM-gldN in F. johnsoniae, are co-transcribed and are absolutely required for the T9SS function in both bacteria (Vincent et al., 2017). It has been proposed that the PorK-PorLPorM-PorN complex form the T9SS core, and that the GldL-GldM inner-membrane complex could act as a rotary motor that energizes the system (Sato et al., 2010; Vincent et al., 2017). We recently solved the structure of the GldM periplasmic domain (Leone et al., 2018) and, in order to collect more structural information on the potentially new rotary motor, we intended to solve the structure of the GldL cytoplasmic domain. Facing unsuccessful crystallization, we decided to use nanobodies as crystallization chaperones. Nanobodies correspond to the variable domain (VHH) from heavy-chainonly immunoglobulins from Camillidae and are valuable tools in structural biology (Desmyter et al., 2015). Indeed, their use was decisive in the structure resolution of the PorM periplasmic domain (Duhoo et al., 2017; Leone et al., 2018). Using the nanobody platform of our laboratory, two nanobodies were 
raised against the GldL cytoplasmic domain; the interaction with their target was characterized and their crystal structures were solved.

\section{Results}

\subsection{Nanobodies generation and binding characterization}

The recombinant GldL cytoplasmic domain (residues 59-215, GldLc) was produced in E. coli, purified to homogeneity, and used to immunize a llama. Several strong GldLc binders were identified after three rounds of panning using phage display coupled to ELISA. Two nanobodies (called hereafter 7BNW and 7BNP) were selected according to their high affinity for GldLc and their sequence differences in the variable regions (also called the complementarity-determining regions, CDRs) (Fig1). Their CDR1s and CDR2s have the same length, but 7BNP CDR3 is one residue longer. 7BNW and 7BNP were produced and purified, and their interaction with GldLc was characterized. 7BNW and 7BNP both bind to GldLc in the nanomolar range (Table1).

\subsection{Nanobody structures}

In addition to serve as crystallization chaperones, nanobodies can be used to solve the structure of the complex with the protein of interest by molecular replacement. We recently used this strategy to solve the structure of the PorM periplasmic N-terminal domain. Thus, as no structural homolog is available for GldLc, we crystallized 7BNW and 7BNP, and we solved their structures by molecular replacement. 7BNW crystals belong to the $\mathrm{P}_{5}$ space group and diffracted to $2.59 \AA$. Four molecules are present in the asymmetric unit, but 7BNW is monomeric in solution at the crystallization concentration (Fig2). The three N-terminal residues, as well as the five C-terminal His residues from the His 6 tag could not be modelled due to weak electron density. As expected, 7BNW adopts the classical immunoglobulin fold with two $\beta$-sheets, each composed of four and five antiparallel $\beta$-strands: $\beta 1-\beta 3-\beta 7-\beta 8$ and $\beta 4$ $\beta 5-\beta 6-\beta 9-\beta 10$. The second and last $\beta$-strands $\beta 2$ and $\beta 11$ lie along one edge of the sandwich, and these strands extend to form a parallel- $\beta$ interaction insuring the proper closure of the $\beta$-sandwich (Fig3). The 
four molecules in the asymmetric unit are very similar, with an r.m.s.d. ranging from 0.30 to $0.85 \AA$ over $\mathrm{C}_{\alpha}$ atoms; the differences arise from the CDRs that display only slightly different conformations.

7BNP crystals belong to the $\mathrm{C} 2$ space group and diffracted to $1.67 \AA$, with one molecule in the asymmetric unit. The three $\mathrm{N}$-terminal residues, as well the $\mathrm{C}$-terminal $\mathrm{His}_{6}$ tag residues and residues at the beginning of CDR1 (residues 25-30, FigS1) could not be modelled due to weak electron density. Interestingly, 7BNP displays a domain-swapped structure: the C-terminal residues (residues 101-119) are exchanged with a symmetric molecule, which reconstitutes an immunoglobulin fold (Fig4). The hinge loop of this domain-swapping corresponds to the CDR3 (residues 98-100e). Indeed, instead of folding back on itself, the CDR3 rather projects toward the second protomer to form an 'arm in arm' dimer. The extended, helical conformation of the CDR3 is stabilized by two cation- $\pi$ interactions on both sides of the $\alpha$-helix of both protomers, that involve the 7NBP CDR3 specific Trp100a of one protomer, and the conserved Arg95 from the other protomer. The end of the CDR3 is locked to the second protomer by reconstituting the conserved double cation- $\pi$ interaction involving Trp103 of one protomer, and Lys45 and Tyr91 from the second protomer, thus positioning the swapped C-terminus to reconstitute the second protomer $\beta$-sandwich. While in the classical nanobody fold the C-terminus $\beta$ strand $\beta 11$ and the short $N$-terminal $\beta$-strand $\beta 2$ are stabilized by $\beta$-sheet interactions, no such secondary elements are present in 7NBP N- and C-termini, reflecting a disturbed conformation of these regions that could weaken the interactions between the C-terminus and the core of the nanobody.

The domain-swapping results in an intricate dimer with a buried interaction surface of $2670 \AA^{2}$ for each monomer (on a total surface of $8376 \AA^{2}$ ), which represents $32 \%$ of their molecular surface. SEC-MALS analysis carried out with 7BNP at the crystallization concentration $\left(60 \mathrm{mg}^{\mathrm{m}} \mathrm{mL}^{-1}\right)$ revealed a molar mass of $\approx 13.8 \mathrm{kDa}$, which corresponds to a monomer (Fig 2). However, as the sample is diluted during the experiment, a concentration dependant dimerization cannot be ruled out. Remarkably, 7BNP elutes well before $7 \mathrm{BNW}$, despite a smaller measured molar mass $(\approx 13.8 \mathrm{kDa}$ and $\approx 14.7 \mathrm{kDa}$ for $7 \mathrm{BNP}$ and $7 \mathrm{BNW}$, respectively; Fig2). As nanobodies are not supposed to interact with the SEC column matrix, this observation suggests that the 7BNP molecular shape is larger than a classical nanobody fold, which 
could be due to the partial unfolding of the 7BNP C-terminus favoured by a weak interaction network with the core of the protein

\section{Concluding remarks}

In the context of the structural studies we carry out on the T9SS, we produced the GldL cytoplasmic domain to solve its crystal structure. As no crystal could be obtained, we decided to generate nanobodies that can be used as crystallization chaperones, and that can help to solve the structure of the complex with the target by molecular replacement. Two nanobodies, 7BNW and 7BNP, were selected and produced. Their nanomolar-range interaction with GldL was characterized by BLI, and their crystal structures were solved. They both adopt the classical immunoglobulin fold with two anti-parallel $\beta$ sheets. Interestingly, 7BNP presents a domain-swapped dimeric structure, with $19 \mathrm{C}$-terminal swapped residues, and the CDR3 acting as the hinge loop. Similar domain-swapping was previously observed for the nanobody VHH-R9, raised against the RR6 red dye hapten (Spinelli et al., 2004). VHH-R9 harbors the shortest CDR3 among camelid nanobodies (six residues), and the seven $\mathrm{N}$-terminal residues (including the first $\beta$-strand $\beta 1$ ) were proteolytically cleaved during crystallization. It was proposed that the VHH-R9 domain-swapping was driven by the strain induced by the short CDR3 together with the lability of the $\mathrm{C}$-terminus that is induced by the absence of the stabilizing $\mathrm{N}$-terminal $\beta$-strand $\beta 1$. Similarly, 7BNP harbors a potentially labile C-terminus with the propensity to unfolding, as suggested by the SEC profiles. On the other hand, the quite long and flexible 7BNP CDR3 (13 residues, comprising four glycine residues) can be stabilized in an extended, protruding conformation through symmetric interactions with a mate molecule, which could further favor the C-terminus swapping.

The 7BNP domain-swapping, with the CDR3 as the hinge loop, raises the question as to whether 7BNP could still act as a crystallization chaperone for GldL. Indeed, the CDR3 is generally important for antigen binding, and its domain-swapping induced conformation as observed in the crystal might be not compatible with GldL interaction. However, domain-swapping is a slow kinetics process that probably takes place during crystallogenesis, when high concentration conditions promote molecular contacts. Given the nanomolar affinity between 7BNP and GldL as measured by BLI, we can expect that the 
complex could be purified at moderate concentration and further concentrated for crystallization trials without formation of 7BNP dimers. Large scale production of GldL-nanobody complexes for crystallization is under way.

\section{Material and Methods}

\subsection{Cloning and production of the GIdL cytoplasmic domain}

The sequence of the cytoplasmic GldL domain corresponding to residues 59-215 (GldLc) was amplified from Flavobacterium johnsoniae cDNA (ATCC17061, Leibniz Institute DSMZ) and was cloned into the pLIC03 vector (kindly provided by BioXtal; unpublished work) using the primers 5'CCGAGAACCTGTACTTCCAATCAGAACCAGTTGAGGATGAATTAG

5'CGGAGCTCGAATTCGGATCCTTATTATCCTTTGTTACTCATTGCAG (sequences annealing on the gldL gene are italicized). The pLIC03 vector is derived from the pET28a+ expression vector in which a cassette coding for a His 6 tag and a Tobacco Etch Virus (TEV) protease cleavage site followed by the $s a c B$ suicide gene flanked by $B s a$ I restriction sites was introduced downstream of the ATG start codon.

GldLc was produced in T7 E. coli cells (NEB) cultured in TB medium at $37^{\circ} \mathrm{C}$ until the $\mathrm{OD}_{600 \mathrm{~nm}}$ reached 0.6-0.8. Then, $1 \mathrm{mM}$ isopropyl- $\beta$-thio-galactoside (IPTG) was added to induce the expression of the protein and the temperature was decreased to $17^{\circ} \mathrm{C}$ for an additional $18 \mathrm{~h}$. The cells were harvested by centrifugation at $4000 \mathrm{rpm}$ for $15 \mathrm{~min}$ at $4^{\circ} \mathrm{C}$ and resuspended in $50 \mathrm{mM}$ Tris $\mathrm{pH} 8.0,300 \mathrm{mM} \mathrm{NaCl}$, $10 \mathrm{mM}$ imidazole (buffer A) supplemented with $0.1 \mathrm{mg} \mathrm{mL}^{-1}$ lysozyme, $1 \mathrm{mM}$ phenylmethylsulfonyl fluoride (PMSF). After freezing $\mathrm{ON}$ at $-80^{\circ} \mathrm{C}$, the pellet was thawed and incubated with $20 \mu \mathrm{g} \cdot \mathrm{mL}^{-1}$ DNAse I and $\mathrm{MgSO}_{4}$ for $30 \mathrm{~min}$ at $4{ }^{\circ} \mathrm{C}$. The cells were lysed by sonication and the insoluble pellet was removed by centrifugation at $14000 \mathrm{~g}$ for $30^{\prime}$ at $4^{\circ} \mathrm{C}$. GldLc was purified from the soluble fraction by metal ion affinity chromatography using a $5 \mathrm{~mL}$ HisTrap crude (GE Healthcare) $\mathrm{Ni}^{2+}$ - chelating column equilibrated in buffer A. GldLc was eluted with buffer A supplemented with 500mM imidazole. The pool containing the purified GldLc was incubated ON with 1:10(w:w) TEV protease to cleave the His 6 tag, and concomitantly dialyzed against buffer A to remove the imidazole. The flowthrough from a 
second nickel-affinity chromatography that contains the cleaved GldLc was collected, and the protein was further purified by size exclusion chromatography on a Hiload 16/60 Superdex 75 prep grade column (GE Healthcare) equilibrated in 10mM Hepes pH7.5, 200mM NaCl.

\subsection{Generation and production of llama nanobodies}

Immunization of one llama (Llama glama from Ardèche lamas France) was achieved with five injections of $1 \mathrm{mg}$ purified GldLc in $10 \mathrm{mM}$ Hepes $\mathrm{pH} 7.5,200 \mathrm{mM} \mathrm{NaCl}$. The selection and screening of nanobodies was performed as described previously (Duhoo et al., 2017) by the Nabgen Technology platform (https://nabgen.org/). After sequence analysis, two different nanobodies (7BNW and 7BNP) were chosen for further characterization. The expression and purification of 7BNW and 7BNP was performed as described previously (Conrath et al., 2009). Briefly, the nanobodies were cloned in pHEN6 vector in fusion with pelB sequence and His 6 tag at the N- and C-termini, respectively, purified from the periplasmic fraction of WK6 cells culture by nickel-affinity chromatography, and further purified by size exclusion chromatography in $10 \mathrm{mM}$ Hepes pH7.5, $200 \mathrm{mM} \mathrm{NaCl}$.

\subsection{Size Exclusion Chromatography-Multi Angle Light Scattering (SEC-MALS)}

For the SEC-MALS analysis, purified 7BNW and 7BNP were concentrated to 24 and $60 \mathrm{mg} \cdot \mathrm{mL}^{-1}$, respectively. Size exclusion chromatography was carried out on an Ultimate 3000 HPLC system (Fischer Scientific) using a Superdex 200 Increase 10/300 GL column (GE Healthcare) equilibrated in $10 \mathrm{mM}$ Hepes $\mathrm{pH} 7.5$ and $200 \mathrm{mM} \mathrm{NaCl}$ at a flow of $0.6 \mathrm{ml} / \mathrm{min}$. Detection was performed using an eight-angle light-scattering detector (DAWN8, Wyatt Technology) and a differential refractometer (Optilab, Wyatt Technology).

\subsection{Bio-layer interferometry (BLI)}

BLI assays were performed as described previously (Duhoo et al., 2017) using an Octet Red96 (Sartorius). Biotinylated 7BNW and 7BNP were loaded on streptavidin biosensor tips (Sartorius) at $5 \mu \mathrm{g} . \mathrm{mL}^{-1}$. The association of each nanobody with various concentration of GldLc $(9.4,32.5,75,150$, 300 and $600 \mathrm{nM}$ ) was recorded for a period of $600 \mathrm{~s}$, followed by dissociation in kinetic buffer (KB; 
Sartorius; PBS, $0.01 \%$ BSA, $0.002 \%$ Tween 20 and $0.005 \%$ sodium azide) for $900 \mathrm{~s}$. Complete dissociation of the complex was reached by three cycles of tips regeneration/neutralization $(5 \mathrm{~s}$ in $10 \mathrm{mM}$ glycine $\mathrm{pH} 1.7$ followed by $5 \mathrm{~s}$ in $\mathrm{KB}$ ). Control experiments were run to check that there was no nonspecific interaction between the analyte (GldLc) and control biosensors (loaded with no protein and blocked with biocytin). The curve-fitting 1:1 binding model was used to measure the constants with the Octet Red system software (v.7.1).

\subsection{Crystallization, data collection and processing}

Crystallization screens using the purified 7BNW $\left(24 \mathrm{mg} \cdot \mathrm{mL}^{-1}\right)$ and 7BNP $\left(60 \mathrm{mg} \cdot \mathrm{mL}^{-1}\right)$ were performed by the sitting-drop vapor diffusion method at $293 \mathrm{~K}$ in 96 -well Swissci-3 plates with Stura Footprint (Molecular Dimensions), Wizard I and II (Rigaku) and Structure I and II (Molecular Dimensions) screens. The reservoirs of the Swissci-3 plates were filled by a TECAN pipetting robot, and the nanodrops were dispensed by a Mosquito robot (TTP Labtech) by mixing different volumes $(100,200$ and 300nl) of protein solution and 100nl precipitant solution. 7BNW crystals were obtained in $100 \mathrm{mM}$ sodium acetate $\mathrm{pH} 4.5,200 \mathrm{mM}$ calcium acetate and 30\% (v/v) PEG 400, and 7BNP crystals were obtained in $100 \mathrm{mM}$ Tris $\mathrm{pH} 8.5$ and 25\% $(w / v)$ PEG 3350. Crystals were mounted in cryo-loops (Hampton CrystalCap Magnetic) and were soaked for few seconds in crystallization solution supplemented with $20 \%(\mathrm{v} / \mathrm{v})$ ethylene glycol before being flash-cooled in a nitrogen-gas stream at 100 K using a cryocooling device (Oxford Cryosystems).

Diffraction data were collected on beamline Proxima-1 at SOLEIL synchrotron (Saint-Aubin, France), and were processed with the XDS package (Kabsch, 2010). The structure of 7BNW and 7BNP were solved by molecular replacement using MOLREP (Vagin \& Teplyakov, 2010). The PDB entries with the highest sequence similarity with 7BNW and 7BNP were used as starting models (1T2J and1 KXQ, respectively). Refinement was performed with autoBUSTER (Blanc et al., 2004), and the structures were corrected with COOT (Emsley \& Cowtan, 2004). For 7BNW, last refinement steps were performed with REFMAC5 (Murshudov et al., 2011) with the twin refinement option. Model 
validations were performed with MolProbity (Chen et al., 2010). Data collection and refinement statistics are given in Table 2.

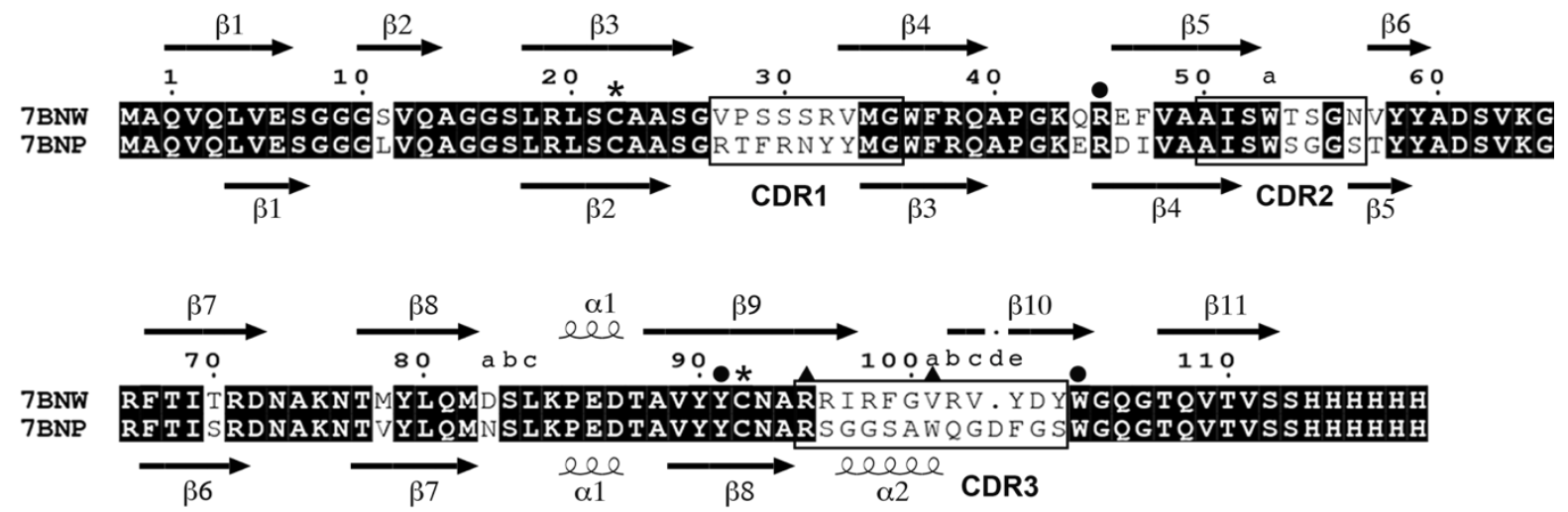

Figure 1 Sequence alignment of 7BNW and 7BNP.

Displayed are the sequences of 7BNW and 7BNP as expressed and crystallized. Secondary structure elements from 7BNW and 7BNP structures are displayed above and below the alignment, respectively. The CDR1, CDR2 and CDR3 sequences are boxed; the cysteine residues are denoted by an asterisk; the residues involved in the 7BNP CDR3 specific and the conserved cation- $\pi$ interactions, as identified by the CaPTURE program (Gallivan \& Dougherty, 1999), are denoted by black triangles and spheres, respectively. Kabat numbering is used (Kabat et al., 1991). The sequence alignment was generated by Multalin (Corpet, 1988), and processed by ESPript (Robert \& Gouet, 2014). 


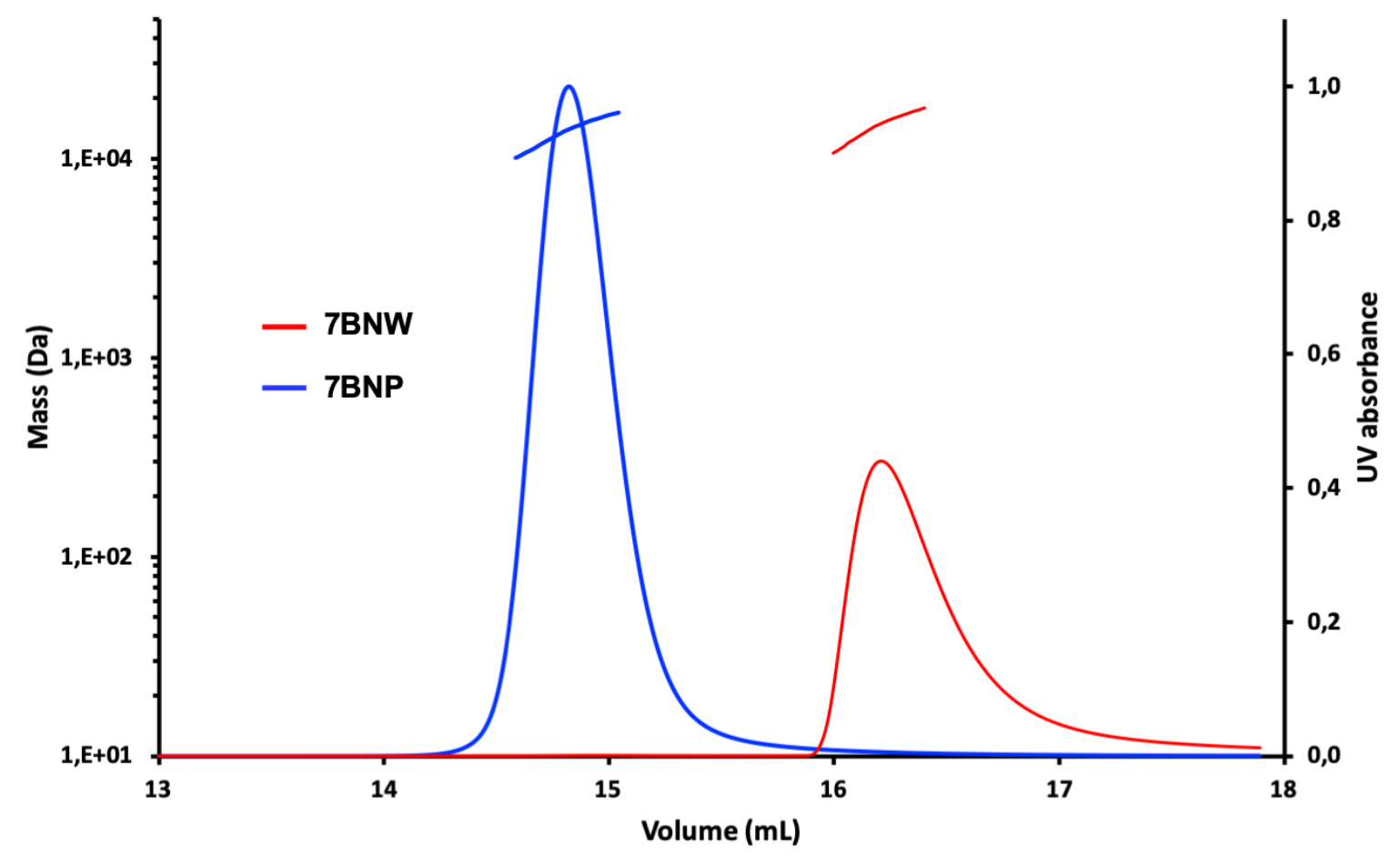

Figure 2 Size Exclusion Chromatography-Multi Angle Light Scattering (SEC-MALS) analysis of 7BNW and 7BNP.

Chromatograms of the purified 7BNW (red) and 7BNP (blue). The measured molar masses are $\approx 14.7 \mathrm{kDa}$ and $\approx 13.8 \mathrm{kDa}$ for $7 \mathrm{BNW}$ and $7 \mathrm{BNP}$, respectively. 


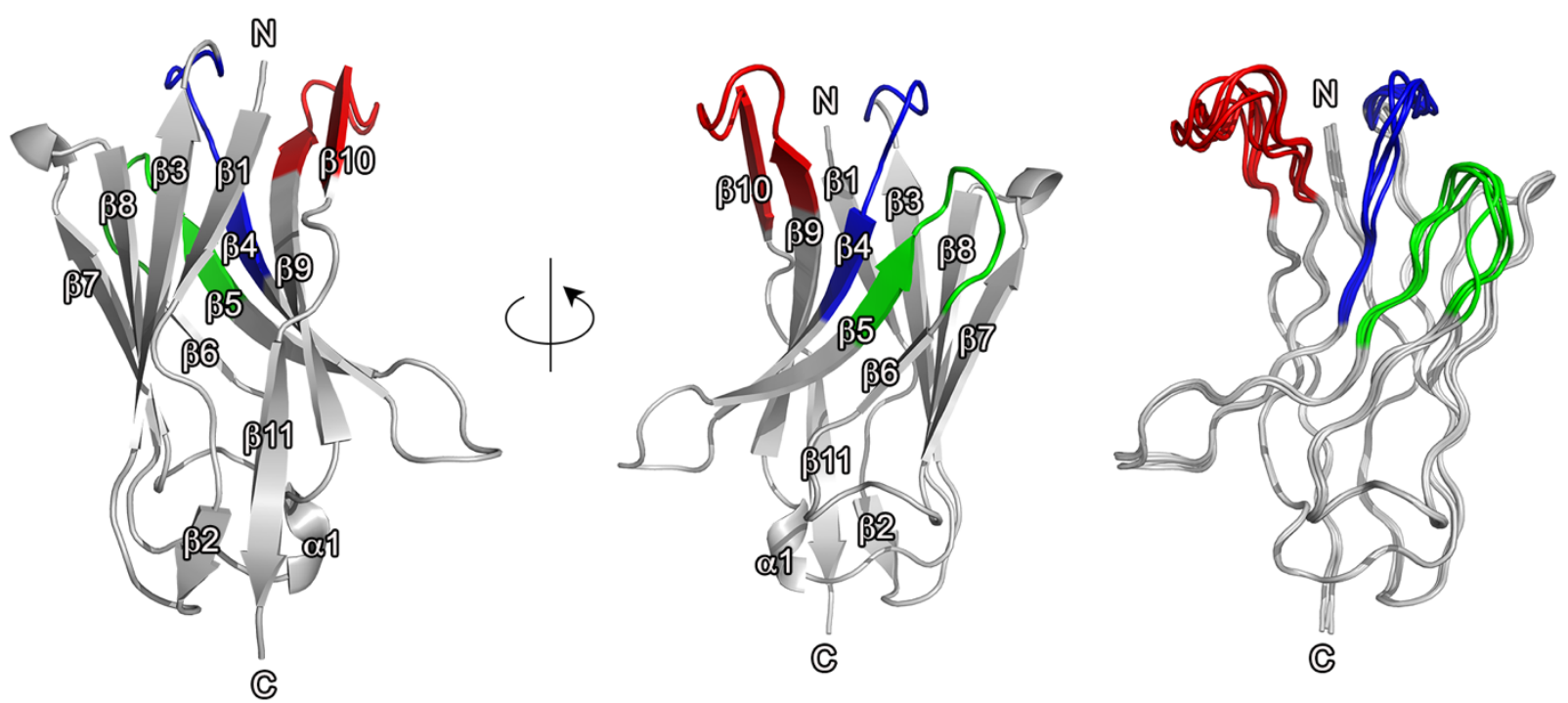

Figure 3 Crystal structure of 7BNW.

Left and middle: only the molecule A is displayed for clarity purpose; right: the four molecules present in the asymmetric unit are superimposed.

The CDR1, CDR2 and CDR3 according to Kabat numbering (Kabat et al., 1991) are represented in blue, green and red, respectively. The $\mathrm{N}$ - and $\mathrm{C}$-terminal extremities are labelled $\mathrm{N}$ and $\mathrm{C}$, respectively. The picture was generated by Pymol (The PyMOL Molecular Graphics System, Schrödinger, LLC). 


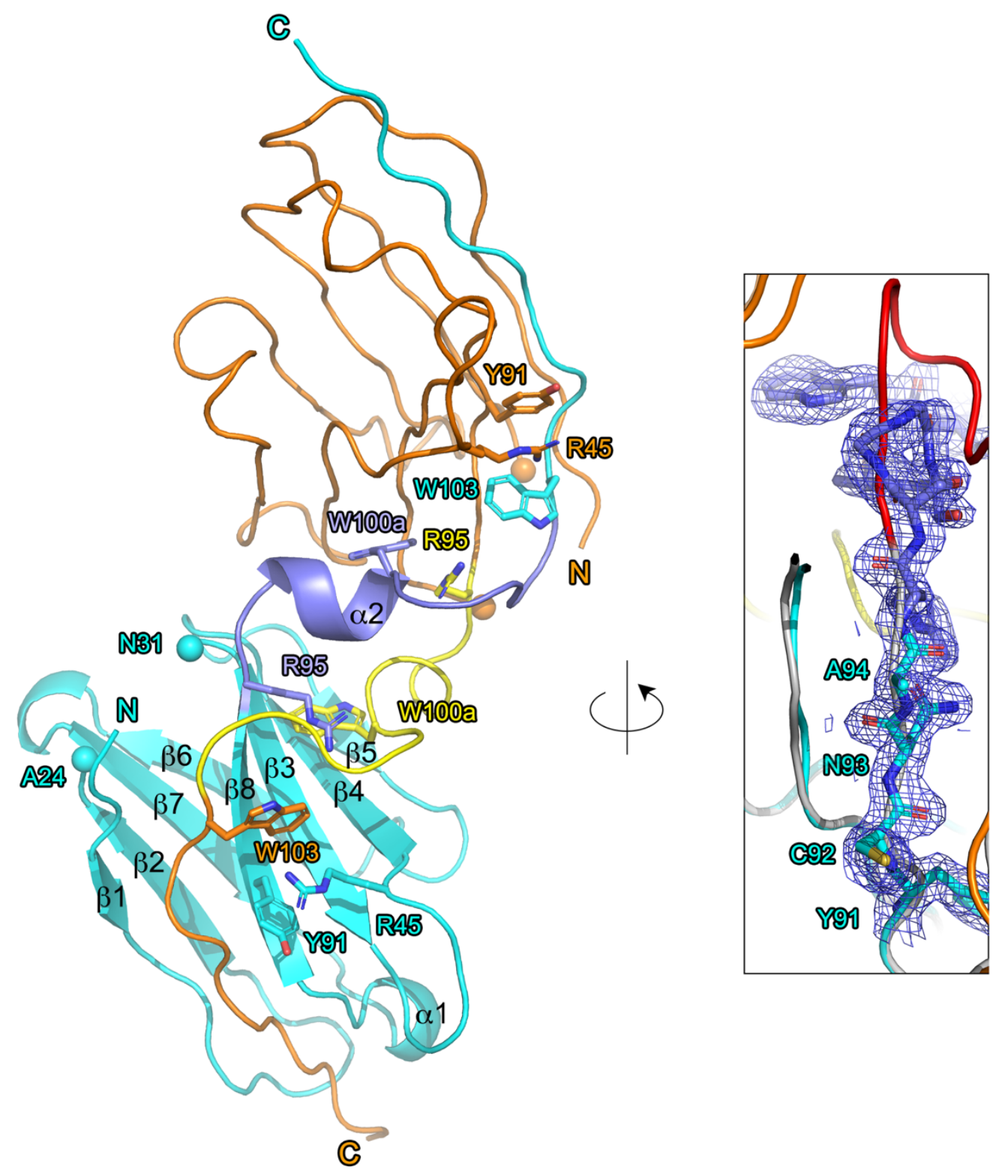

Figure 4 Domain-swapped dimer in the 7BNP crystal.

7BNP is displayed in cyan, with the CDR3 in blue; the domain-swapped symmetric mate is displayed in orange, with the CDR3 in yellow, as worm diagram for clarity purpose. The residues involved in cation- $\pi$ interactions are displayed in stick format; the residues at both sides of the CDR1 part that is not visible in the electron density map (Ala24 and Asn31) are displayed in sphere format; the N-and C-terminal extremities are labelled $\mathrm{N}$ and $\mathrm{C}$, respectively.

Enclosed is a close-up view of the domain-swapping hinge. The CDR3 with residues before (Ala94, Asn93, Cys92 and Tyr91), are displayed in stick format, and the corresponding electron density map (contoured at $1 \sigma$ ) is displayed in blue. Superimposed 7BNW is displayed in light grey with the CDR3 in red. 7BNP and 7BNW are displayed as worm diagram for clarity purpose. 
The picture was generated by Pymol (The PyMOL Molecular Graphics System, Schrödinger, LLC).

Table 1 Kinetic and thermodynamic parameters of the interactions between 7BNW and 7BNP with the GldL periplasmic domain (Gldc).

\begin{tabular}{lllll}
\hline & $\mathrm{K}_{\mathrm{D}}(\mathrm{nM})$ & $\mathrm{K}_{\text {ass }} \mathrm{M}^{-1} \mathrm{~s}^{-1}$ & $\mathrm{~K}_{\text {diss }}\left(\mathrm{s}^{-1}\right)$ & full $\mathrm{Chi}^{2}-$ full $\mathrm{R}^{2}$ \\
GIdLc / 7BNW & $8.1 \pm 0.35$ & $4.4 \times 10^{5}$ & $3.5 \times 10^{-4}$ & $3.87-0.93$ \\
GIdLc / 7BNP & $32 \pm 0.36$ & $8.5 \times 10^{4}$ & $2.7 \times 10^{-3}$ & $7.44-0.98$ \\
\hline
\end{tabular}

Table 2 Data collection and refinement statistics of 7BNW and 7BNP.

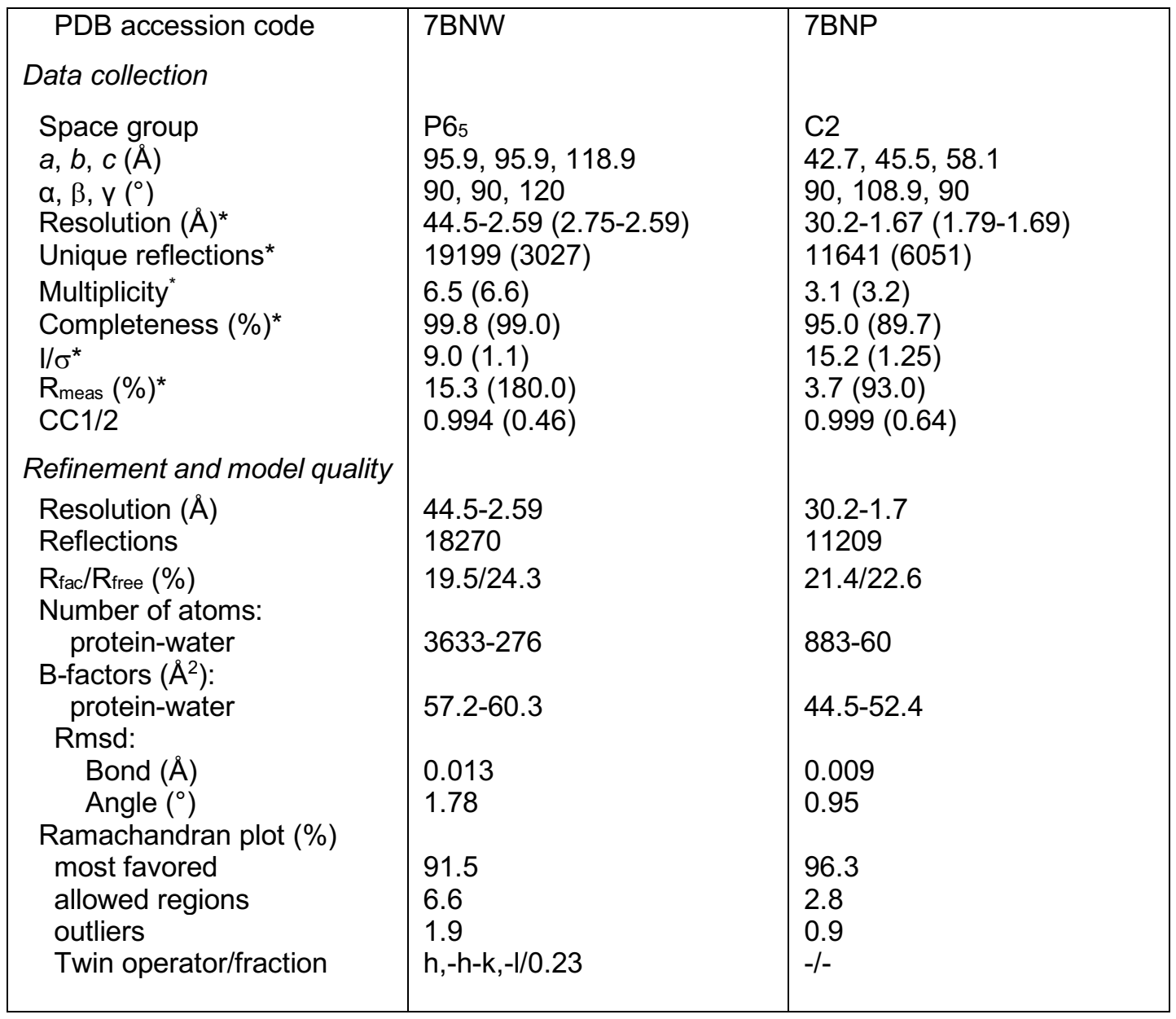

* Values in parentheses are for the highest-resolution shell. 
Acknowledgements We would like to thank the SOLEIL synchrotron for beamline allocation.

\section{References}

Blanc, E., Roversi, P., Vonrhein, C., Flensburg, C., Lea, S. M., \& Bricogne, G. (2004). Acta Crystallogr. D. Biol. Crystallogr. 60, 2210-2221.

Chen, V. B., Arendall, W. B., Headd, J. J., Keedy, D. A., Immormino, R. M., Kapral, G. J., Murray, L. W., Richardson, J. S., \& Richardson, D. C. (2010). Acta Crystallogr. Sect. D Biol. Crystallogr. 66, $12-21$.

Conrath, K., Pereira, A. S., Martins, C. E., Timóteo, C. G., Tavares, P., Spinelli, S., Kinne, J., Flaudrops, C., Cambillau, C., Muyldermans, S., et al. (2009). Protein Sci. 18, 619-628. Corpet, F. (1988). Nucleic Acids Res.

Desmyter, A., Spinelli, S., Roussel, A., \& Cambillau, C. (2015). Curr. Opin. Struct. Biol. 32, 1-8. Duhoo, Y., Roche, J., Trinh, T. T. N., Desmyter, A., Gaubert, A., Kellenberger, C., Cambillau, C., Roussel, A., \& Leone, P. (2017). Acta Crystallogr. Sect. F Struct. Biol. Commun. 73, 286-293.

Emsley, P. \& Cowtan, K. (2004). Acta Crystallogr D Biol Crystallogr. 60, 2126-2132.

Gallivan, J. P. \& Dougherty, D. A. (1999). Proc. Natl. Acad. Sci. U. S. A. 96, 9459-9464.

Kabsch, W. (2010). Acta Crystallogr. D. Biol. Crystallogr. 66, 133-144.

Lasica, A. M., Ksiazek, M., Madej, M., \& Potempa, J. (2017). Front. Cell. Infect. Microbiol. 7, 215. Leone, P., Roche, J., Vincent, M. S., Tran, Q. H., Desmyter, A., Cascales, E., Kellenberger, C., Cambillau, C., \& Roussel, A. (2018). Nat. Commun. 9, 429.

McBride, M. J. \& Zhu, Y. (2013). J. Bacteriol. 195, 270-278.

Murshudov, G. N., Skubák, P., Lebedev, A. A., Pannu, N. S., Steiner, R. A., Nicholls, R. A., Winn, M. D., Long, F., \& Vagin, A. A. (2011). Acta Crystallogr. D. Biol. Crystallogr. 67, 355-367. Robert, X. \& Gouet, P. (2014). Nucleic Acids Res. 42,.

Sato, K., Naito, M., Yukitake, H., Hirakawa, H., Shoji, M., McBride, M. J., Rhodes, R. G., \& 
Nakayama, K. (2010). Proc. Natl. Acad. Sci. U. S. A. 107, 276-281.

Spinelli, S., Desmyter, A., Frenken, L., Verrips, T., Tegoni, M., \& Cambillau, C. (2004). FEBS Lett. 564, 35-40.

Vagin, A. \& Teplyakov, A. (2010). Acta Crystallogr. D. Biol. Crystallogr. 66, 22-25.

Veith, P. D., Glew, M. D., Gorasia, D. G., \& Reynolds, E. C. (2017). Mol. Microbiol. 106, 35-53.

Vincent, M. S., Canestrari, M. J., Leone, P., Stathopoulos, J., Ize, B., Zoued, A., Cambillau, C.,

Kellenberger, C., Roussel, A., \& Cascales, E. (2017). J. Biol. Chem. jbc.M116.765081. 